\title{
Altered Cardiolipin Metabolism is Associated with Cardiac Mitochondrial Dysfunction in Pulmonary Vascular Remodeled Perinatal Rat Pups
}

Laura K. Cole ${ }^{1,2}$, Genevieve C. Sparagna ${ }^{3}$, Vernon W. Dolinsky ${ }^{1,2}$ and Grant M. Hatch ${ }^{1,2}$ Affiliations: ${ }^{1}$ Department of Pharmacology and Therapeutics, and ${ }^{2}$ Diabetes Research Envisioned and Accomplished in Manitoba (DREAM) Theme, Children's Hospital Research Institute of Manitoba, Rady Faculty of Health Sciences, University of Manitoba, Winnipeg, Canada. ${ }^{3}$ Department of Medicine, Division of Cardiology, University of Colorado Anschutz Medical Center, Aurora, Colorado, USA.

"To whom correspondence should be addressed:

Dr. Grant M. Hatch, 501C-JBRC, Children's Hospital Research Institute of Manitoba Winnipeg, Manitoba, Canada, R3E 3P4, Telephone: (204) 789- 3405, Fax: (204) 7893932, Email: ghatch@chrim.ca

\section{Paper: 4939 words (including references)}

\section{Tables: 0}

Figures: 6

\section{Supplementary Figures: 3}

Category: Basic Science

Funding support statement: Supported by Heart and Stroke Foundation of Canada, Canadian Institutes of Health Research, Natural Sciences and Engineering Research Council, Children's Hospital Research Institute of Manitoba, University of Manitoba.

Disclosure statement: The authors have no conflicts of interest.

Patient consent: Not required for this study

Author contributions: LKC, VWD \& GMH were responsible for conceptual design and writing and editing of the manuscript. LKC, GCS performed experiments. All authors reviewed the manuscript. 


\section{Impact statement}

- Phospholipid metabolism was examined in pulmonary vascular remodeling in perinatal rat pups.

- Pulmonary vascular remodeling was induced in utero by treating pregnant dams with hypoxia and indomethacin at 19-21 days of gestation.

- The offspring exhibited altered pulmonary arterial remodeling with subsequent cardiac hypertrophy, ventricular dysfunction, cardiac myocyte mitochondrial dysfunction with altered fatty acid utilization.

- In addition, the offspring exhibited elevated cardiolipin, lysocardiolipin and phosphatidylethanolamine content which may potentially contribute to the cardiac mitochondrial dysfunction. 


\section{Abstract}

Background: Pulmonary vascular remodeling (PVR) in utero results in the development of heart failure (HF). The alterations that occur in cardiac lipid and mitochondrial bioenergetics during the development of in utero PVR was unknown.

Methods: PVR was induced in pups in utero by exposure of pregnant dams to indomethacin and hypoxia. Cardiac lipids, echocardiographic function and cardiomyocyte mitochondrial function were subsequently examined.

Results: Perinatal rat pups with PVR exhibited elevated left and right cardiac ventricular internal dimensions and reduced ejection fraction and fractional shortening compared to controls. Cardiac myocytes from these pups exhibited increased glycolytic capacity and glycolytic reserve compared to controls. However, respiration with glucose as substrate was unaltered. Fatty acid oxidation and ATP-insensitive respiration were increased in isolated cardiac myocytes from these pups compared to controls indicating mitochondrial dysfunction. Although abundance of mitochondrial respiratory complexes were unaltered, increased trilinoleoyl-lysocardiolipin levels in these pups was observed. A compensatory increase in both cardiolipin $(\mathrm{CL})$ and phosphatidylethanolamine (PE) content were observed due to increased synthesis of these phospholipids.

Conclusion: Alterations in cardiac cardiolipin and phospholipid metabolism in PVR rat pups is associated with the mitochondrial bioenergetic and cardiac functional defects observed in their hearts.

\section{Word count: 193}

Running Title: Altered cardiolipin metabolism in pulmonary vascular remodeled rat pup hearts 


\section{Introduction}

Pulmonary hypertension of the newborn is a failure of normal pulmonary vascular relaxation after birth, with incidence of up to 6 per 1000 live births and $10-30 \%$ mortality (1-3). It is a significant cause of heart failure (HF) in healthy newborn infants. In healthy term infants it is caused by perinatal hypoxia, inflammation or direct lung injury. These infants develop the HF rapidly within a week and HF becomes the main limiting factor for their survival. A number of studies in human and animal models have demonstrated alterations in mitochondrial bioenergetics during the development of HF (4). Several of these studies have implicated alterations in the mitochondrial phospholipid cardiolipin $(\mathrm{CL})$ as a contributor to the mitochondrial dysfunction. $\mathrm{CL}$, the signature phospholipid of mitochondria, is essential for mitochondrial morphology, bioenergetics, dynamics, and signaling pathways (5-11). However, limited information exists on the changes in cardiac function and phospholipid composition that occur during in utero pulmonary vascular remodeling (PVR). Previously we demonstrated that newborn piglets exposed to a hypoxic environment for 3 days developed alterations in $C L$ and reduced mitochondrial respiratory chain dysfunction during the development of a right ventricular hypertrophy (12).

In this study, we utilized a unique gestational rat model PVR to examine how cardiac phospholipid metabolism, cardiac morphology and mitochondrial function are impacted by PVR. We show for the first time that $C L$ and phosphatidylethanolamine (PE) levels are elevated in the hearts of PVR rat pups and that this is accompanied by an 
bioRxiv preprint doi: https://doi.org/10.1101/2021.10.12.464115; this version posted October 12, 2021. The copyright holder for this preprint (which was not certified by peer review) is the author/funder. All rights reserved. No reuse allowed without permission.

accumulation of lysocardiolipin (LysoCL) and mitochondrial bioenergetic and cardiac dysfunction. 


\section{Materials and Methods}

\section{Animals}

This study was performed with approval of the University of Manitoba Animal Policy and Welfare Committee which adheres to the principles for biomedical research involving animals developed by the Canadian Council on Animal Care and the Council for International Organizations of Medical Sciences. This study is reported in accordance with ARRIVE guidelines. All animals were maintained in an environmentally controlled facility $\left(22^{\circ} \mathrm{C}, 37 \%\right.$ humidity, $12 \mathrm{~h}$ light/dark cycle) with free access to food and water.

PVR was induced in perinatal rats by treating pregnant dams during 19-21 days of gestation with hypoxia and indomethacin (single ip dose $0.5 \mathrm{mg} / \mathrm{Kg}$ in sterile PBS pH 7.4) as described $(13,14)$. Timed pregnant rats at 18 days gestation were utilized. One set of pregnant rats were housed normally (Control, single ip dose sterile PBS) as control animals, a second set of pregnant rats were indomethacin-treated as above (Indo), while a third set of pregnant rats were indomethacin-treated and then housed in a hypoxic environment (12\% oxygen) (PVR). This was accomplished by housing rats in a plexiglass chamber and the chamber was maintained at $12 \%$ oxygen using a nitrogen washout system. Medical grade compressed oxygen (12\% oxygen balance Nitrogen, Welder's Supply) was used in combination with medical grade nitrogen to adjust chamber oxygen levels to $12 \%$ using a gas analyzer (Radiometer $A B L 700$ series). Compressed gas flowed continuously into the chamber at a rate of $1 \mathrm{~L} / \mathrm{min}$ for 3 days. Chamber oxygen levels, flow rates and temperatures were monitored every $24 \mathrm{~h}$ at a minimum. On the fourth day all animals were sacrificed and caesarean section was performed to remove the pups. Prior to sacrifice fetal echocardiographic parameters were determined as described 
below. After removal by caesarean section the pups were then weighed, measured for length and the heart, liver and lung removed and weighed. Histology of pulmonary arteries was performed as previously described (12). In some experiments, the heart was freeze dried and weighed. In other experiments, freshly isolated hearts were used for preparation of mitochondrial fractions or isolation of cardiac myocytes as outlined below.

\section{In vivo echocardiography}

Transthoracic echocardiography was performed on dams mildly anesthetized with $1-1.5 \%$ isoflurane, $1 \mathrm{~L} / \mathrm{min}$ oxygen as previously described (15). Each rat was placed on a heated ECG platform to maintain body temperature and obtain fetal heart measurements. A Vevo 2100 High resolution imaging system equipped with a $30-\mathrm{MHz}$ transducer (Visual Sonics, Toronto) was used to visualize the fetal hearts. Measurements obtained included interventricular septum diastolic (IVSd), interventricular septum systolic (IVSs), left ventricle internal dimension diastolic (LVIDd), left ventricle internal dimension systolic (LVIDs), left ventricular wall diastolic (LVWd), intraventricular septum end systole (LVSs), ejection fraction (EF), fractional shortening (FS), right ventricular wall diastolic (RVWd), right ventricular wall systolic (RVWs), right ventricle internal dimension diastolic (RVIDd) and right ventricle internal dimension systolic (RVIDs).

\section{Cardiac myocyte preparation and radiolabeling experiments}

Cardiac myocytes were prepared from the hearts of control and PVR pups as described (16). The protocol allows for rat cardiomyocytes to be cultured for up to $72 \mathrm{~h}$ at $37^{\circ} \mathrm{C}$ in $5 \% \mathrm{CO}_{2}$ without significant change in phenotype (17). Enough hearts were collected to yield approximately $15-20$ million cardiac myocytes sufficient to plate 15-20 
x 35mm dishes (1 million/dish, Corning Primeria ${ }^{\mathrm{TM}}$ ). Isolated cardiac myocytes were incubated with $0.1 \mathrm{mM}\left[1,3-{ }^{3} \mathrm{H}\right]$ glycerol $(2 \mu \mathrm{Ci} /$ dish, Perkin Elmer $)$ or $0.1 \mu \mathrm{M}\left[1-{ }^{14} \mathrm{C}\right]$ linoleic acid $\left(2 \mu \mathrm{Ci} / \mathrm{dish}\right.$, Perkin Elmer) bound to albumin (1:1 molar ratio) or $0.1 \mu \mathrm{M}\left[1-{ }^{14} \mathrm{C}\right]$ oleic acid $(2 \mu \mathrm{Ci} /$ dish Perkin Elmer) bound to albumin (1:1 molar ratio) for up to $360 \mathrm{~min}(6 \mathrm{~h})$ and radioactivity incorporated into phospholipids determined as previously described (18).

\section{Respiratory function analysis}

The oxygen consumption rate (OCR) was measured from isolated cardiac myocytes of control and PVR pups ( $1 \times 10^{5} /$ well, coated with fibronectin) using a Seahorse XF24 Bioscience instrument (18). XF assay media contained either $1 \mathrm{mmol} / \mathrm{L}$ pyruvate and $25 \mathrm{mmol} / \mathrm{L}$ glucose for glucose metabolism or $1 \mathrm{mmol} / \mathrm{L}$ pyruvate, $2.5 \mathrm{mmol} / \mathrm{L}$ glucose, $0.5 \mu \mathrm{mol} / \mathrm{L}$ carnitine, and $0.175 \mathrm{mmol} / \mathrm{L}$ palmitate-BSA for fatty acid (FA) metabolism. Basal oxygen consumption was considered to be the basal respiration sensitive to inhibition by $1 \mu \mathrm{mol} / \mathrm{L}$ antimycin $\mathrm{A}$ plus $1 \mu \mathrm{mol} / \mathrm{L}$ rotenone. ATP-sensitive oxygen consumption was inhibited by $1 \mu \mathrm{mol} / \mathrm{L}$ oligomycin, and ATP-insensitive respiration (heat) was the remaining proportion of basal oxygen consumption. Maximal oxygen consumption was achieved with $1 \mu \mathrm{mol} / \mathrm{L}$ carbonyl cyanide 4-(trifluoromethoxy) phenylhydrazone. Fatty acid-dependent respiration was measured as the difference in oxygen consumption measured in the presence of $40 \mu \mathrm{mol} / \mathrm{L}$ etomoxir and vehicle (water). The XF glycolysis stress test kit (Aligent) was used for glycolysis analysis. Briefly, cells are cultured in the absence of glucose followed by the sequential addition of glucose $(10 \mathrm{mM})$, oligomycin $(3 \mu \mathrm{M})$ and 2-DG $(1 \mathrm{M})$. Where glycolysis is measured following glucose addition (glucose - 2-DG), glycolytic capacity measured following oligomycin 
addition (oligomycin - 2-DG), and glycolytic capacity the difference between them (glycolytic capacity-glucose).

\section{Western blot analysis of mitochondrial respiratory subunits}

Cardiac mitochondria were isolated from control or PVR hearts using the MITOISO1 Mitochondria Isolation Kit (Sigma) as previously described (15). Mitochondrial protein $(7.5 \mu \mathrm{g})$ from was separated on the Bio-Rad mini gel electophoresis system by SDS-PAGE (12\% acrylamide) as previously described (15). Western blot antibody cocktail (Abcam) contained (55 kDa, anti-ATP synthase subunit ATP5a, Complex V; 47 kDa, anti-complex III subunit core 2 UQCRC2; 35 kDa, anti-complex IV subunit MTCO1; $30 \mathrm{kDa}$, complex II subunit SDHB; and $20 \mathrm{kDa}$, complex I subunit NDUFB8). Adult rat mitochondrial protein was added for comparative control and $\alpha$-tubulin (Cell Signaling) was used as the loading control. Proteins were visualized by chemiluminescence using the ECL Western blotting detection system (Amersham).

\section{Lipid analysis}

The level of the major phospholipids from cardiac tissue homogenates including phosphatidylcholine (PC), PE and CL were determined by HPLC separation followed by lipid phosphorus assay $(19,20)$. Molecular species of CLs and lysoCLs were quantitated from tissue homogenates by HPLC coupled to electrospray ionization mass spectrometry (21). Cardiac cholesterol, cholesterol ester and triacylglycerol were determined by HPLC as previously described (15).

\section{Statistical analysis}


Data are expressed as means \pm standard error of the mean (SEM). Comparisons between control and PVR offspring were determined using 1-way analysis of variance using Tukey post-hoc analysis. For each measurement, the offspring were derived from multiple litters. A probability $p$ value of $<0.05$ was considered significant. 


\section{Results}

PVR was induced in perinatal rats by treating pregnant dams during 19-21 days of gestation with hypoxia and indomethacin $(13,14)$. Lung histology analysis of newborn rats revealed classic PVR in pups from the hypoxic-indomethacin treated group compared to control including increased medial thickness in the larger pulmonary arteries with no changes in their external diameter (Fig. 1A-D). Thus, we established this model of PVR in fetal rats $(13,14)$. Reduction in body weight and length of newborn PVR pups were accompanied by reductions in both lung and liver weight compared to control (Fig. 2A-D). Dried heart weight/body weight ratio was elevated in PVR pups compared to control (Fig. $2 \mathrm{E})$ and this was accompanied alterations in cardiac structural and functional parameters (Fig. 3A-C). Specifically, in the left ventricle elevations in IVSd, IVSs, LVWd and LVSs were accompanied by a reduction in EF and FS. In addition, right ventricle dimensions including RVWd and RVWs were elevated in PVR pups compared to control. Thus, the PVR rat hearts exhibited cardiac hypertrophy and mechanical dysfunction.

We examined if substrate utilization was altered in isolated cardiac myocytes from rats in which PVR was induced during gestation. Oxygen consumption rate (OCR) was unaltered in PVR pups compared to control when glucose was used as substrate (Suppl. Fig. 1A). Interestingly, glycolytic capacity and glycolytic reserve were increased in PVR pups compared to control (Fig. 4A). In addition, when palmitate was used as substrate, an increase in basal OCR was observed which was blocked by incubation with the carnitine palmitoyltransferase-1 inhibitor etomoxir (Fig. 4B). The increase in fatty acid (FA) oxidation was accompanied an increase in ATP-sensitive oxygen consumption (Fig. 4C) and ATP-insensitive respiration (Fig. 4D) in PVR pups compared to control. Maximum 
OCR was unaltered in cardiac myocytes between control and PVR pups (Fig. 4E). Thus, the increase in ATP-insensitive respiration indicated a dysfunction in efficiency of mitochondrial FA oxidation in cardiac myocytes from PVR rat pups.

We next examined the pool sizes of the three major phospholipids in hearts from control and PVR pups. Phospholipid analysis revealed that there was no alteration in the levels of phosphatidylcholine (PC) but striking increases in both cardiolipin (CL) and phosphatidylethanolamine (PE) in hearts of PVR pups compared to control (Fig. 5A-C). The observed reduction in cardiac PC/PE ratio was consistent with that seen in pressureinduced heart failure (22). In addition, the levels of cardiac cholesterol, cholesterol ester and triacylglycerol were unaltered (Suppl. Fig. 2A-C). Since CL, and specifically tetralinoleoylcardiolipin $\left(\mathrm{L}_{4} \mathrm{CL}\right)$, are required for optimal cardiac mitochondrial bioenergetic function (23), we examined the molecular species composition of CL. Elevations in 1442, 1448 and 1472 species of $C L$ appeared to be responsible for the observed increase in CL in PVR pups (Fig. 5D). The highest being linoleate containing ${ }_{4} \mathrm{CL}$ (1448). In isolated cardiac myocytes from PVR pups the increase in $\mathrm{L}_{4} \mathrm{CL}$ was due to an increase in $\left[1-{ }^{-14} \mathrm{C}\right]$ linoleate incorporation into $\mathrm{CL}$ indicating increased synthesis from linoleate (Fig $6 \mathrm{~A})$. In contrast, de novo synthesis of $\mathrm{CL}$ from $\left[1,3^{-}{ }^{3} \mathrm{H}\right]$ glycerol was unaltered in cardiac myocytes from PVR rat pups (Fig. 6B).

Since the increase in $\mathrm{L}_{4} \mathrm{CL}$ did not explain the mitochondrial dysfunction, we examined the levels of cardiac mitochondrial respiratory complexes. No alteration in the abundance of mitochondrial complexes was observed in PVR pups compared to controls (Suppl. Fig. 1B). LysoCL accumulation is known to cause mitochondrial bioenergetic dysfunction (24). We observed elevated trilinoleoyl-lysoCL ( $\mathrm{L}_{3}$-lysoCL) species in hearts 
of PVR pups compared to controls (Fig. 5E). Thus, an elevation in linoleate containing L3lysoCL species could be linked to the observed cardiac mitochondrial bioenergetic dysfunction in PVR rat pups.

The reason for the elevation in PE was also examined. Incorporation of radioactivity into $\mathrm{PE}$ from $\left[1,3-{ }^{3} \mathrm{H}\right]$ glycerol was unaltered in isolated cardiomyocytes from PVR rat pups indicating that de novo synthesis was unaltered (Fig. 6B). To further confirm this, synthesis of PE from serine and ethanolamine were examined. There was no alteration in synthesis of PE from $\left[{ }^{3} \mathrm{H}\right]$ ethanolamine nor synthesis of $\mathrm{PE}$ or phosphatidylserine from $\left[{ }^{3} \mathrm{H}\right]$ serine in cardiac myocytes from PVR rat pups (Suppl. Fig $3 A, B)$. In contrast, the increase in $P E$ was due to an increase in $\left[1-{ }^{14} C\right]$ oleate incorporation into PE by $6 \mathrm{~h}$ of incubation indicating increased synthesis from oleate (Fig $6 C)$. 


\section{Discussion}

In this study, we examined how altered cardiac CL and phospholipid metabolism and mitochondrial dysfunction are associated with the perinatal cardiac pathophysiology of PVR in perinatal rat pups. We show that PVR in perinatal rat pups result in cardiac hypertrophy and ventricular dysfunction, cardiac myocyte mitochondrial dysfunction with altered FA substrate utilization and elevated $\mathrm{CL}, \mathrm{L}_{3}$-LysoCL and $\mathrm{PE}$ which may contribute, in part, to the cardiac mitochondrial dysfunction.

In this study, we have characterized for the first time the cardiac lipid alterations that occur in pups of the in utero hypoxia and indomethacin-induced fetal PVR rat model $(13,14)$. There are several reports of indomethacin induced pulmonary hypertension in human newborns mediated by premature constriction of the fetal ductus arteriosus (2528). In addition, indomethacin-mediated closure of the ductus arteriosus in fetal rats was shown to result in early-onset right ventricular hypertrophy (29). Perinatal pulmonary hypertension in rats is known to permanently modify the pulmonary vasculature $(30,31)$. Consistent with this we observed that pulmonary arteries from our pups exhibited increased medial thickness in the larger pulmonary arteries with no changes in their external diameter. Associated with the pulmonary arterial modification was a cardiac hypertrophy characterized by elevation in right ventricle dimensions including RVWd and RVWs. In addition, left ventricle elevations in IVSd, IVSs, LVWd and LVSs, and reduction in EF and FS were observed. The elevation in right ventricular dimensions were consistent with that previously reported in this model of PVR (32).

Alteration in substrate utilization is a hallmark of cardiac dysfunction in HF (33). Studies in animal models and in human pulmonary arterial hypertension suggest that 
there is increased glycolysis and a metabolic shift from oxidative mitochondrial metabolism to the less energy efficient glycolytic metabolism $(34,35)$. Although basal OCR was unaltered in isolated cardiac myocytes of PVR pups when glucose was used as substrate, we observed increased glycolytic capacity and glycolytic reserve suggesting the potential for increase in glucose utilization for ATP synthesis. When palmitate was used as substrate an increase in FA oxidation was accompanied by an increase in ATPinsensitive respiration in isolated cardiac myocytes of PVR pups.

Pressure induced cardiac failure in rodents is known to result in increased PE levels and cardiac dysfunction (22). In addition, right ventricular pressure overload in adult rats induced by 12 weeks pulmonary arterial banding resulted in elevations in PE (34). We observed elevated cardiac PE levels, with no changes in PC, in hearts of perinatal PVR rat pups. The observed reduction in cardiac PC/PE ratio is consistent with that seen in pressure-induced heart failure (22). The increase in PE was not due to increased de novo synthesis or decarboxylation from phosphatidylserine since PE synthesis from radiolabeled glycerol, ethanolamine or serine was unaltered. In contrast, pulse-labeling with $\left[{ }^{1-14}\right]$ oleate revealed increased synthesis of PE from oleate was responsible for the accumulation of PE in isolated cardiac myocytes from PVR rat pups.

A key question is whether alteration in CL levels actually contribute to the development of pediatric HF (36). A number of adult animal and human studies have indicated that reduced $\mathrm{CL}$ and $\mathrm{L}{ }_{4} \mathrm{CL}$ accompany the development of $\mathrm{HF}(4,37,38)$. The reduction in cardiac $\mathrm{CL}$ in many early studies of adult $\mathrm{HF}$ in rodents can be attributed to the feeding of defined diets which may subject these animals to accelerated HF (39). The animals used in our study were harvested by caesarean section and were not subjected 
to maternal feeding. It was recently demonstrated that the relative percentage of $\mathrm{L}_{4}-\mathrm{CL}$ was preserved in pediatric human congenital single ventricle heart disease samples relative to biventricular controls (40). In addition, differences in CL content were not observed in induced pluripotent stem cell cardiac myocytes prepared from control and pediatric dilated cardiomyopathy with ataxia syndrome patients (41). Another study observed that the CL profiles in pediatric HF were unique from those in adults and the authors of this study hypothesized that end-stage pediatric heart failure adaptive mechanisms to preserve $\mathrm{L}_{4} \mathrm{CL}$ content may be intact to a greater degree than that seen in adult heart failure (42). Our results support the above hypothesis as we observed increased $\mathrm{CL}$ and $\mathrm{L}_{4} \mathrm{CL}$ levels in the hearts of caesarean section harvested perinatal PVR rat pups. Although de novo synthesis of $\mathrm{CL}$ from glycerol was unaltered in cardiac myocytes of perinatal PVR rat pups, an increase in $\left[1-{ }^{14} \mathrm{C}\right]$ linoleate incorporation into CL was observed which would explain the accumulation of $\mathrm{CL}$ and $\mathrm{L}_{3}$-lysoCL. The elevation in ${ }_{4} \mathrm{CL}$ might contribute to the observed increase in FA oxidation in isolated cardiac myocytes from these animals. However, the apparent compensatory increase in FA oxidation in isolated cardiac myocytes from PVR rat pups was accompanied by an increased state 4 (oligomycin-inhibited) respiration indicative of an elevated proton leak which likely contributes to the mitochondrial dysfunction.

Accumulation of lysoCL is known to result in mitochondrial bioeneregtic dysfunction by compromising the stability of the protein-dense mitochondrial inner membrane leading to a decrease in optimal respiration (24). Although we observed no alteration in the abundance of individual mitochondrial complexes subunits, the significant accumulation of $\mathrm{L}_{3}$-lysoCL observed in the hearts of perinatal PVR rat pups might 
contribute to the mitochondrial bioenergetic dysfunction through a morphological disturbance of cristae membrane structure.

A limitation of the use of this hypoxia-induced model of PVR is whether the observed effects on metabolism may simply be due to the hypoxia itself. However, while hypoxia may cause a global change in cardiac homeostasis, the perinatal rats used in our study exhibited the cumulative effects of pressure, and the additional increase in cardiac weight was due to the effect of increased afterload. In addition, it is possible that the metabolic effects we observed may be more prominent in the right ventricle than the left ventricle. However, metabolism is also abnormal in the left ventricle in pulmonary arterial hypertension (43). In summary, our data show for the first time that a perturbed CL and PE metabolism is associated with and may contribute, in part, to the mitochondrial bioenergetic and cardiac functional defects observed in the heart of the hypoxia and indomethacin-induced gestational model of perinatal PVR in rats.

\section{Acknowledgements}

The authors wish to thank Marilyne Vandel for technical assistance. LKC was the recipient of a CIHR/HSFC IMPACT Fellowship. GMH is a Canada Research Chair in Molecular Cardiolipin Metabolism. VWD is the Allen Rouse-Manitoba Medical Services Foundation Basic Scientist. This research was supported by an Environments, Genes and Chronic Disease Canadian Institutes for Health Research (CIHR) Team Grant \#144626, the Heart and Stroke Foundation of Canada, the Natural Sciences and Engineering Research Council (NSERC), Children's Hospital Research Institute of Manitoba (CHRIM) and the University of Manitoba Research Grants Program (URGP). 


\section{References}

1. Therese, P. Persistent pulmonary hypertension of the newborn. Paediatr. Respir. Rev. 7, Suppl 1:S175-6 (2006).

2. Vosatka, R.J. Persistent pulmonary hypertension of the newborn. N. Engl. J. Med. 346, 864 (2002).

3. Dakshinamurti, S. Pathophysiologic mechanisms of persistent pulmonary hypertension of the newborn. Pediatr. Pulmonol. 39, 492-503 (2005).

4. Dolinsky, V.W., Cole, L.K., Sparagna, G.C., \& Hatch, G.M. Cardiac mitochondrial energy metabolism in heart failure: Role of cardiolipin and sirtuins. Biochim Biophys Acta. 1861, 1544-1554 (2016).

5. Claypool, S.M., \& Koehler, C.M. The complexity of cardiolipin in health and disease. Trends Biochem. Sci. 37, 32-41 (2012).

6. Falabella, M., Vernon, H.J., Hanna, M.G., Claypool, S.M., \& Pitceathly, R.D.S. Cardiolipin, Mitochondria, and Neurological Disease. Trends Endocrinol. Metab. 32, 224-237 (2021).

7. Ikon, N., \& Ryan, R.O. Barth Syndrome: Connecting Cardiolipin to Cardiomyopathy. Lipids 52, 99-108 (2017).

8. Ren, M., Phoon, C.K., \& Schlame, M. Metabolism and function of mitochondrial cardiolipin. Prog. Lipid Res. 55, 1-16 (2014).

9. Shen, Z., Ye, C., McCain, K., \& Greenberg ML. The Role of Cardiolipin in Cardiovascular Health. Biomed. Res. Int. 2015:891707 (2015).

10.Wasmus, C., \& Dudek, J. Metabolic Alterations Caused by Defective Cardiolipin Remodeling in Inherited Cardiomyopathies. Life (Basel). 10, 277 (2020). 
11.Zegallai, H.M., \& Hatch, G.M. Barth syndrome: cardiolipin, cellular pathophysiology, management, and novel therapeutic targets. Mol. Cell. Biochem. 76, 1605-1629 (2021).

12. Saini-Chohan, H.K., Dakshinamurti, S., Taylor, W.A., Shen, G.X., Murphy, R., Sparagna, G.C., \& Hatch, G.M. Persistent pulmonary hypertension results in reduced tetralinoleoylcardiolipin and mitochondrial complex II + III during the development of right ventricular hypertrophy in the neonatal pig heart. Am. J. Physiol. Heart Circ. Physiol. 301, H1415-24 (2011).

13. Xu XF, Gu WZ, Wu XL, Li RY, Du LZ. Fetal pulmonary vascular remodeling in a rat model induced by hypoxia and indomethacin. J Matern Fetal Neonatal Med. 2011 Jan;24(1):17282

14. Xu XF, Ma XL, Shen Z, Wu XL, Cheng F, Du LZ. Epigenetic regulation of the endothelial nitric oxide synthase gene in persistent pulmonary hypertension of the newborn rat. J Hypertens. 2010 Nov;28(11):2227-35.

15. Cole LK, Mejia EM, Sparagna GC, Vandel M, Xiang B, Han X, Dedousis N, Kaufman BA, Dolinsky VW, Hatch GM. Cardiolipin deficiency elevates susceptibility to a lipotoxic hypertrophic cardiomyopathy. J Mol Cell Cardiol. 2020 Jul;144:24-34

16. Kovacic S, Soltys CL, Barr AJ, Shiojima I, Walsh K, Dyck JR. Akt activity negatively regulates phosphorylation of AMP-activated protein kinase in the heart. $\mathrm{J}$ Biol Chem. 2003 Oct 10;278(41):39422-7.

17. Chan, A.Y., Soltys, C.L., Young, M.E., Proud C.G. and Dyck, J.R. Activation of AMPactivated protein kinase inhibits protein synthesis associated with hypertrophy in the cardiac myocyte. 2004 J Biol Chem 279, 32771-9. 
18. Hatch, G., McClarty, G. Regulation of cardiolipin biosynthesis in H9c2 cardiac myoblasts by cytidine 5'-triphosphate. J Biol Chem. 1996 Oct 18;271(42):25810-6.

19. Cole LK, Mejia EM, Vandel M, Sparagna GC, Claypool SM, Dyck-Chan L, Klein J, Hatch GM. Impaired Cardiolipin Biosynthesis Prevents Hepatic Steatosis and Diet-Induced Obesity. Diabetes. 2016 Nov;65(11):3289-3300.

20. Rouser G, Siakotos AN, Fleischer S. Quantitative analysis of phospholipids by thin-layer chromatography and phosphorus analysis of spots. Lipids. 1, 85-86 (1966).

21.Sparagna, G.C., Johnson, C.A., McCune, S.A., Moore, R.L. \& Murphy, R.C. Quantitation of cardiolipin molecular species in spontaneously hypertensive heart failure rats using electrospray ionization mass spectrometry. J. Lipid Res. 46, 1196-204 (2005)

22. Salatzki J, Foryst-Ludwig A, Bentele K, Blumrich A, Smeir E, Ban Z, Brix S, Grune J, Beyhoff N, Klopfleisch R, Dunst S, Surma MA, Klose C, Rothe M, Heinzel FR, Krannich A, Kershaw EE, Beule D, Schulze PC, Marx N, Kintscher U. Adipose tissue ATGL modifies the cardiac lipidome in pressure-overload-induced left ventricular failure. PLoS Genet 2018 Jan 10;14(1):e1007171.

23. Mejia EM, Cole LK, Hatch GM. Cardiolipin metabolism and the role it plays in heart failure and mitochondrial supercomplex formation. Cardiovasc Hematol Disord Drug Targets. 2014;14(2):98-106.

24. Duncan, AL. Monolysocardiolipin (MLCL) interactions with mitochondrial membrane proteins. Biochem Soc Trans 2020 Jun 30;48(3):993-1004.

25. Lozano González CH, Jiménez PG, Pezzotti MA, Favela EL. Persistent pulmonary hypertension in the newborn caused by prenatal use of prostaglandin inhibitors, (indomethacin). Ginecol. Obstet. Mex. 1980 Aug;48(286):103-110. 
26. Moise Jr KJ, Huhta JC, Sharif DS, Ou CN, Kirshon B, Wasserstrum N, Cano L. Indomethacin in the treatment of premature labor. Effects on the fetal ductus arteriosus. N. Engl. J. Med. 1988 Aug 11;319(6):327-31.

27. Rudolph AM. The effects of nonsteroidal antiinflammatory compounds on fetal circulation and pulmonary function. Obstet Gynecol. $1981 \mathrm{Nov;58(5} \mathrm{Suppl):63S-7S.}$

28. Tarcan A, Gürakan B, Yildirim S, Ozkiraz S, Bilezikçi B. Persistent pulmonary hypertension in a premature newborn after 16 hours of antenatal indomethacin exposure. J. Perinat. Med. 2004;32(1):98-99.

29. Fabris VE, Pato MD, Belik J. Progressive lung and cardiac changes associated with pulmonary hypertension in the fetal rat. Pediatr. Pulmonol. 2001 May;31(5):344-53.

30. Harker LC, Kirkpatrick SE, Friedman WF, Bloor CM. Effects of indomethacin on fetal rat lungs: a possible cause of persistent fetal circulation (PFC). Pediatr. Res. 1981 Feb;15(2):147-51.

31. Herget J, Hampl V, Povýsilová V, Slavík Z. Long-term effects of prenatal indomethacin administration on the pulmonary circulation in rats. Eur Respir J. 1995 Feb;8(2):209-15.

32. Du Y, Fu J, Yao L, Qiao L, Liu N, Xing Y, Xue X. Altered expression of PPAR-y and TRPC in neonatal rats with persistent pulmonary hypertension. Mol Med Rep. 2017 Aug;16(2):1117-1124.

33. Murashige D, Jang C, Neinast M, Edwards JJ, Cowan A, Hyman MC, Rabinowitz JD, Frankel DS, Arany Z. Comprehensive quantification of fuel use by the failing and nonfailing human heart. Science 370, 364-368 (2020)

34. Koop AC, Bossers GPL, Ploegstra MJ, Hagdorn QAJ, Berger RMF, Silljé HHW, Bartelds B. Metabolic Remodeling in the Pressure-Loaded Right Ventricle: Shifts in 
Glucose and Fatty Acid Metabolism-A Systematic Review and Meta-Analysis. J Am Heart Assoc. 2019 Nov 5;8(21):e012086

35. Piao L, Marsboom G, Archer SL. Mitochondrial metabolic adaptation in right ventricular hypertrophy and failure. J Mol Med (Berl). 2010 Oct;88(10):1011-20

36. Fillmore N, Lopaschuk GD. The link between pediatric heart failure and mitochondrial lipids. J Mol Cell Cardiol. 2014 Nov;76:71-2.

37. Sparagna GC, Chicco AJ, Murphy RC, Bristow MR, Johnson CA, Rees ML, Maxey ML, McCune SA, Moore RL. Loss of cardiac tetralinoleoyl cardiolipin in human and experimental heart failure. J Lipid Res. 2007 Jul;48(7):1559-70.

38. Yoshizawa T, Sakurai T, Kamiyoshi A, Ichikawa-Shindo Y, Kawate H, lesato Y, Koyama T, Uetake R, Yang L, Yamauchi A, Tanaka M, Toriyama Y, Igarashi K, Nakada T, Kashihara T, Yamada M, Kawakami H, Nakanishi H, Taguchi R, Nakanishi T, Akazawa $\mathrm{H}$, Shindo T. Novel regulation of cardiac metabolism and homeostasis by the adrenomedullin-receptor activity-modifying protein 2 system. Hypertension. 2013 Feb;61(2):341-51 .

39. Rees, M. L ; Gioscia-Ryan, R. A ; McCune, S. A ; Browder, J. C ; Zachman, D. K ; Chicco, A. J ; Johnson, C. A ; Murphy, R. C ; Moore, R. L ; Sparagna, G. C. The AIN-76A defined rodent diet accelerates the development of heart failure in SHHF rats: A cautionary note on its use in cardiac studies. J. Animal Physiol. Nutr. 2014 Feb;98(1):56-64

40. Garcia AM, McPhaul JC, Sparagna GC, Jeffrey DA, Jonscher R, Patel SS, Sucharov CC, Stauffer BL, Miyamoto SD, Chatfield KC. Alteration of cardiolipin biosynthesis and remodeling in single right ventricle congenital heart disease. Am J Physiol Heart Circ Physiol. 2020 Apr 1;318(4):H787-H800. 
41. Rohani L, Machiraju P, Sabouny R, Meng G, Liu S, Zhao T, Iqbal F, Wang X, Ravandi A, Wu JC, Khan A, Shutt T, Rancourt D, Greenway SC. Reversible Mitochondrial Fragmentation in iPSC-Derived Cardiomyocytes From Children With DCMA, a Mitochondrial Cardiomyopathy. Can J Cardiol. 2020 Apr;36(4):554-563.

42. Chatfield KC, Sparagna GC, Sucharov CC, Miyamoto SD, Grudis JE, Sobus RD, Hijmans J, Stauffer BL. Dysregulation of cardiolipin biosynthesis in pediatric heart failure. J Mol Cell Cardiol. 2014 Sep;74:251-9

43. Ryan JJ, Archer SL. Emerging concepts in the molecular basis of pulmonary arterial hypertension: part I: metabolic plasticity and mitochondrial dynamics in the pulmonary circulation and right ventricle in pulmonary arterial hypertension. Circulation. 2015 May 12;131(19):1691-702. 


\section{Legend to Figures}

Figure 1. Histology of PVR arteries.

Masson's trichrome staining of control (A) and PVR (B) pulmonary arteries. Representative sections are depicted. C. Medial thickness, and D. External diameter of control and PVR pulmonary arteries. The black bar insert represents $50 \mu \mathrm{M}$. Data represent the mean \pm SEM, $n=5$ Control, $n=23 P V R,{ }^{*} p<0.05$.

Figure 2. Body weight, length and organ weight of PVR rats.

Whole body weight $(A)$ and length $(B)$ of control and PVR rat pups. C. Heart weight and dry heart weight/body weight ratio of control and PVR rats. Data represent the mean \pm SEM, $\mathrm{n}=5$ Control, $\mathrm{n}=23$ PVR, ${ }^{*} \mathrm{p}<0.05$.

Figure 3. Echocardiographic parameters of PVR hearts.

Transthoracic echocardiography was performed on control and PVR rats. A. Interventricular septum diastolic (IVSd), interventricular septum systolic (IVSs), left ventricle internal dimension diastolic (LVIDd), left ventricle internal dimension systolic (LVIDs), left ventricular wall diastolic (LVWd), intraventricular septum end systole (LVSs). B. Ejection fraction (EF) and fractional shortening (FS). C. Right ventricular wall diastolic (RVWd), right ventricular wall systolic (RVWs), right ventricle internal dimension diastolic (RVIDd) and right ventricle internal dimension systolic (RVIDs). Data represent the mean \pm SEM, $n=5$ Control, $n=23$ PVR, ${ }^{*} p<0.05$. 
Figure 4. Mitochondrial dysfunction is observed in PVR rats.

A.Glycolysis, glycolytic capacity and glycolytic reserve in control and PVR cardiomyocytes. ECAR, extracellular acidification rate $(\mathrm{mPH} / \mathrm{min} / \mathrm{ug}$ protein). Basal oxygen consumption rate (OCR) (pmol/min/ug protein) (B), ATP-sensitive OCR (pmol/min/ug protein) (C), ATP-insensitive OCR (pmol/min/ug protein) (D) and maximum (Max) OCR (pmol/min/ug protein) (E) in control and PVR cardiomyocytes. V, vehicle; $E$, plus etomoxir; FAO, calculated fatty acid oxidation. Data represent the mean \pm SEM of $\mathrm{n}=3,{ }^{*} \mathrm{p}<0.05$.

Figure 5. PE and CL are elevated in PVR rat hearts.

The content of PC (A), CL (B) and PE (C) and CL molecular species composition (D) and lysoCL molecular species composition $(E)$ were determined in hearts of newborns from control, indomethacin (Indo) or PVR rats. D. the numbers represent individual CL molecular species. E. LLL, L3-lysocardiolipin; LLO, linoleoyl, linoloeyl, oleoyl-lysoCL; LLA, linoleoyl, linoleoyl, arachidonyl-lysoCL. In D and E: Control, black bars; Indo, red bars; $P V R$, green bars. Data represent the mean \pm SEM of $n=3,{ }^{*} p<0.05$.

Figure 6. Synthesis of phospholipids from glycerol, linoleate and oleate in isolated PVR cardiac myocytes.

Isolated control or PVR cardiomyocytes were incubated for up to $360 \mathrm{~min}$ with [1$\left.{ }^{14} \mathrm{C}\right]$ linoleate $(\mathrm{A}),\left[1,3-{ }^{3} \mathrm{H}\right] \mathrm{glycerol}(\mathrm{B})$ or $\left[1-{ }^{14} \mathrm{C}\right]$ oleate $(\mathrm{C})$ and radioactivity incorporated in PC, PE and CL determined. Closed circles, control; Open circles, PVR. Data represent the means \pm SEM of $n=3,{ }^{*} p<0.05$. 


\section{Figure 1}

A

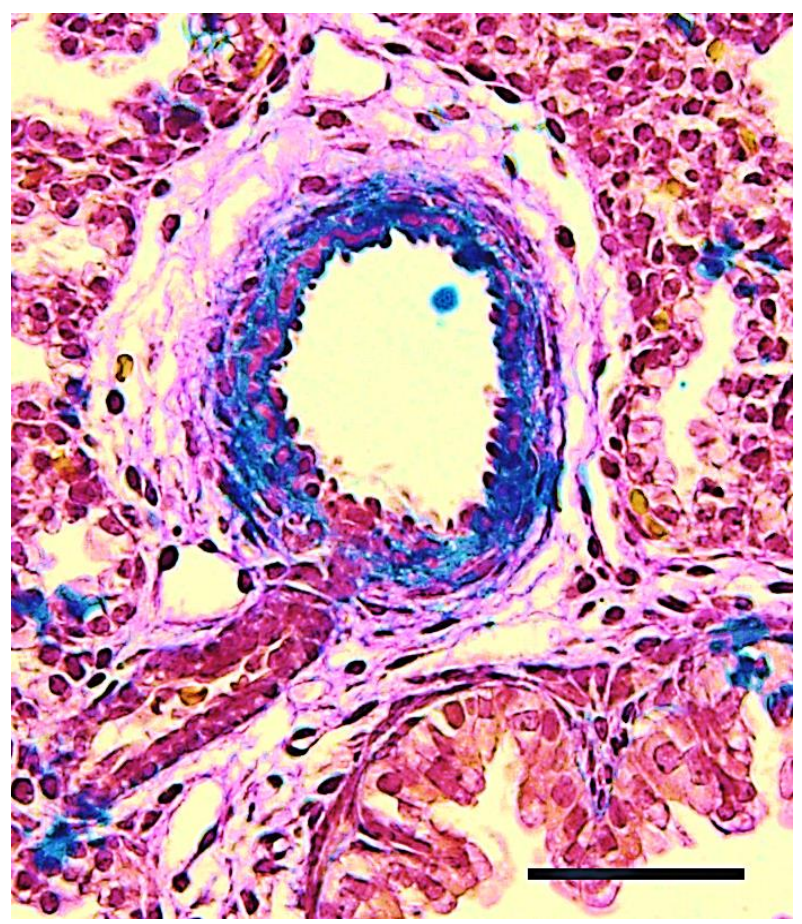

C

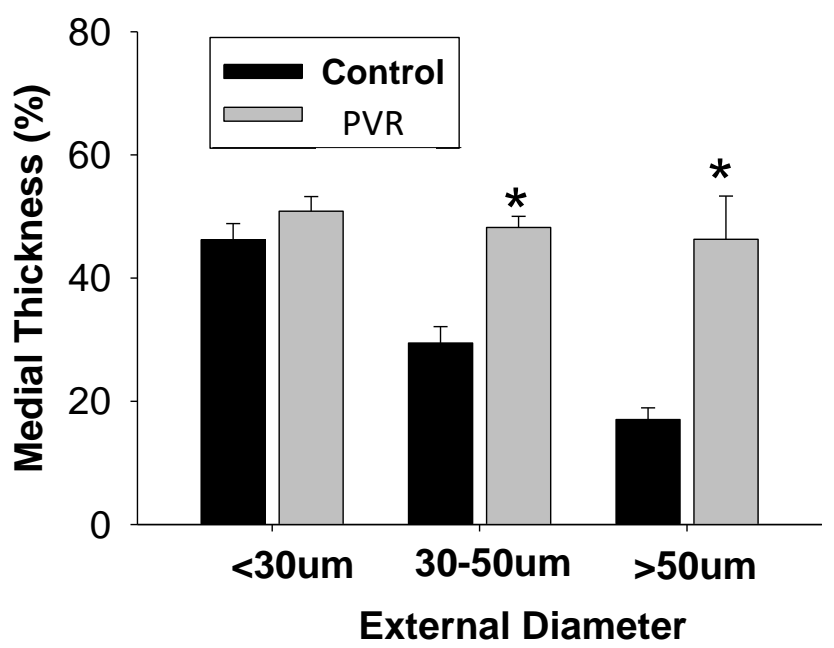

B PVR

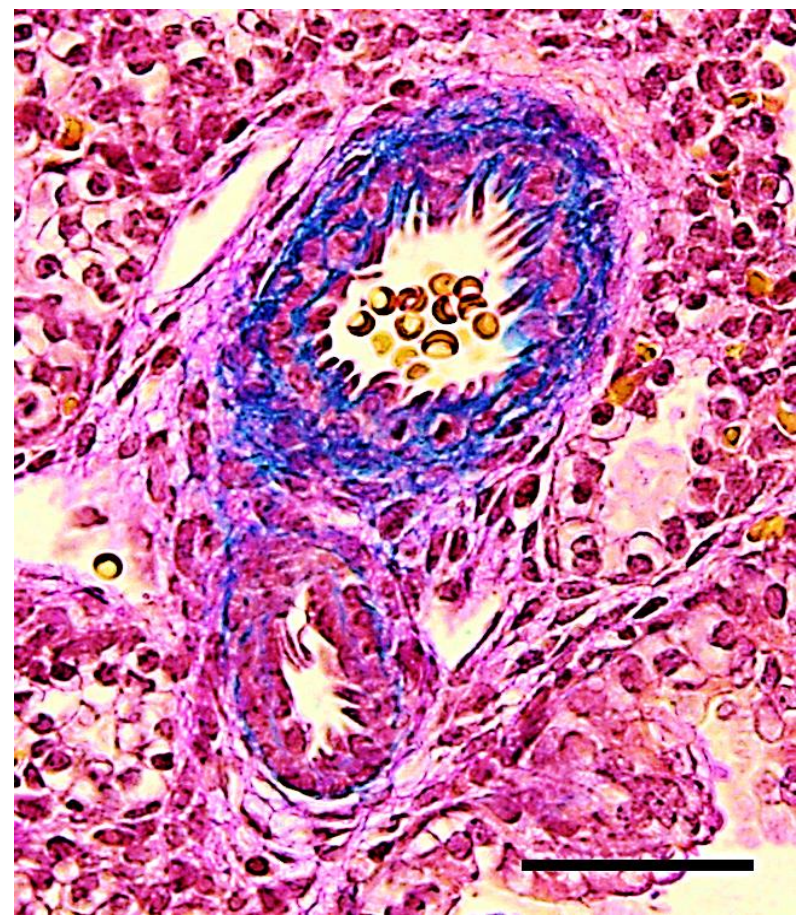

D

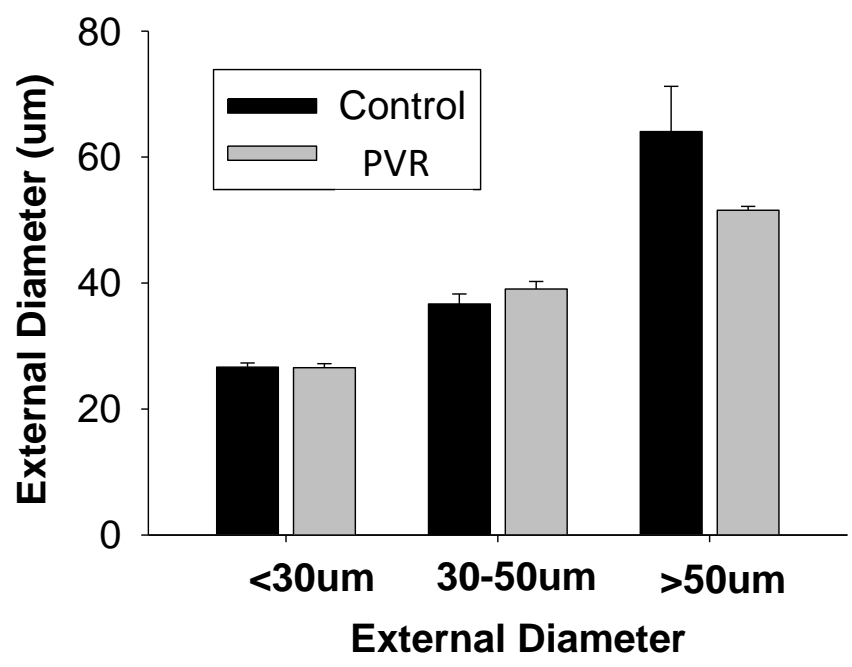




\section{Figure 2}

A

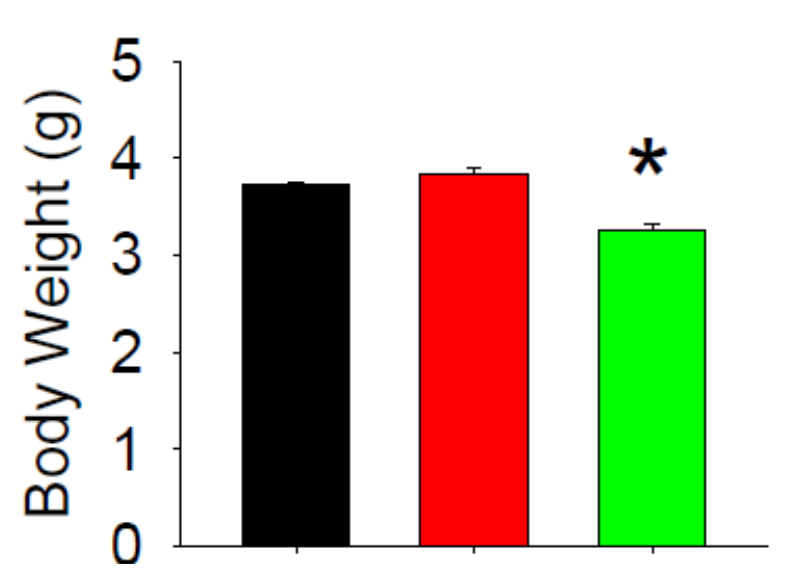

Control Indo PVR

C
B

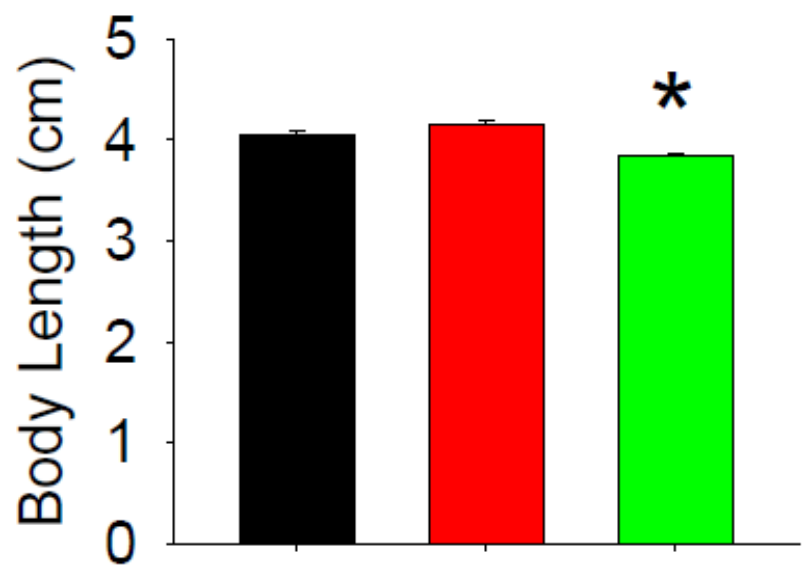

Control Indo PVR

D

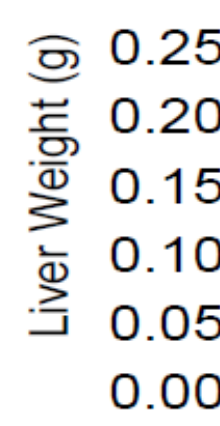

Control Indo PVR

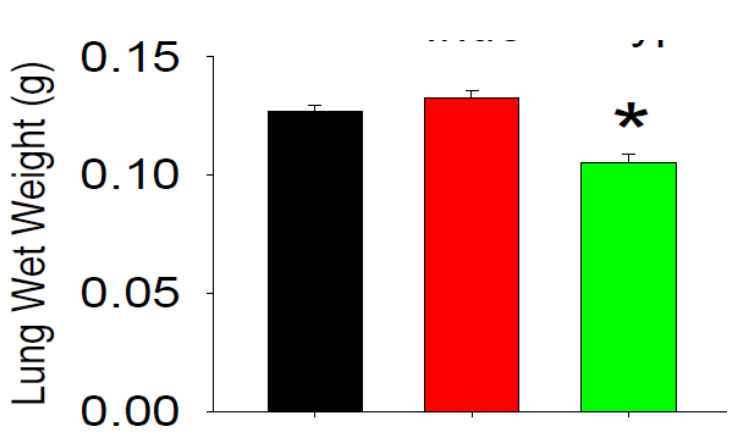

Control Indo PVR

E

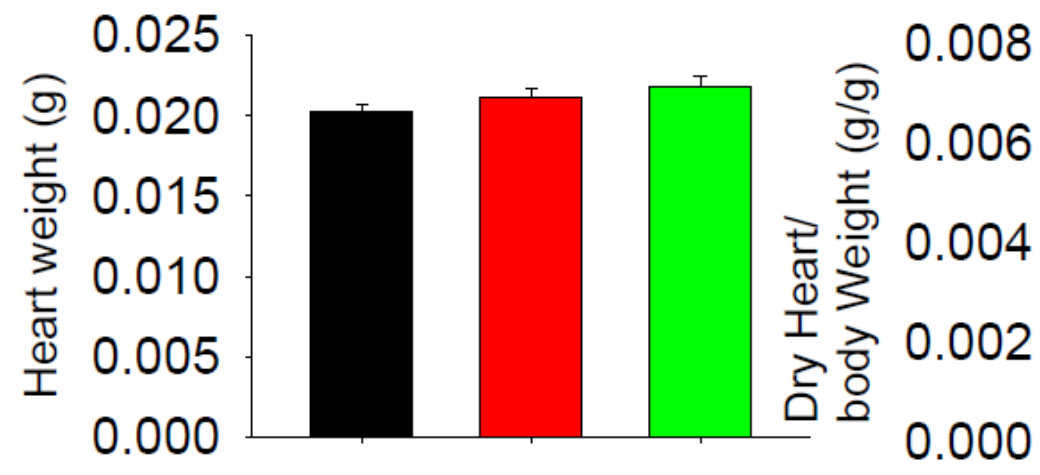

Control Indo PVR

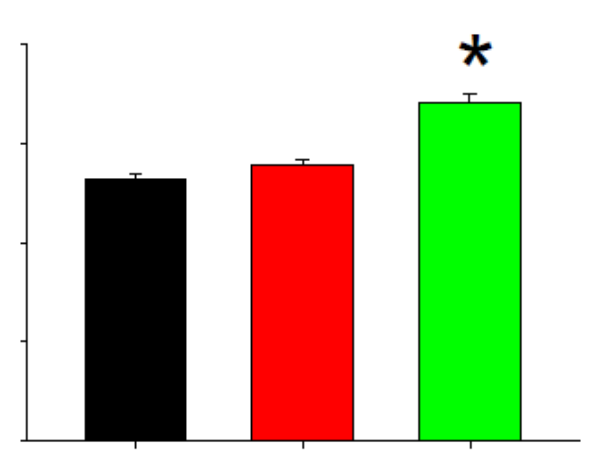

Control Indo PVR 
Figure 3

A

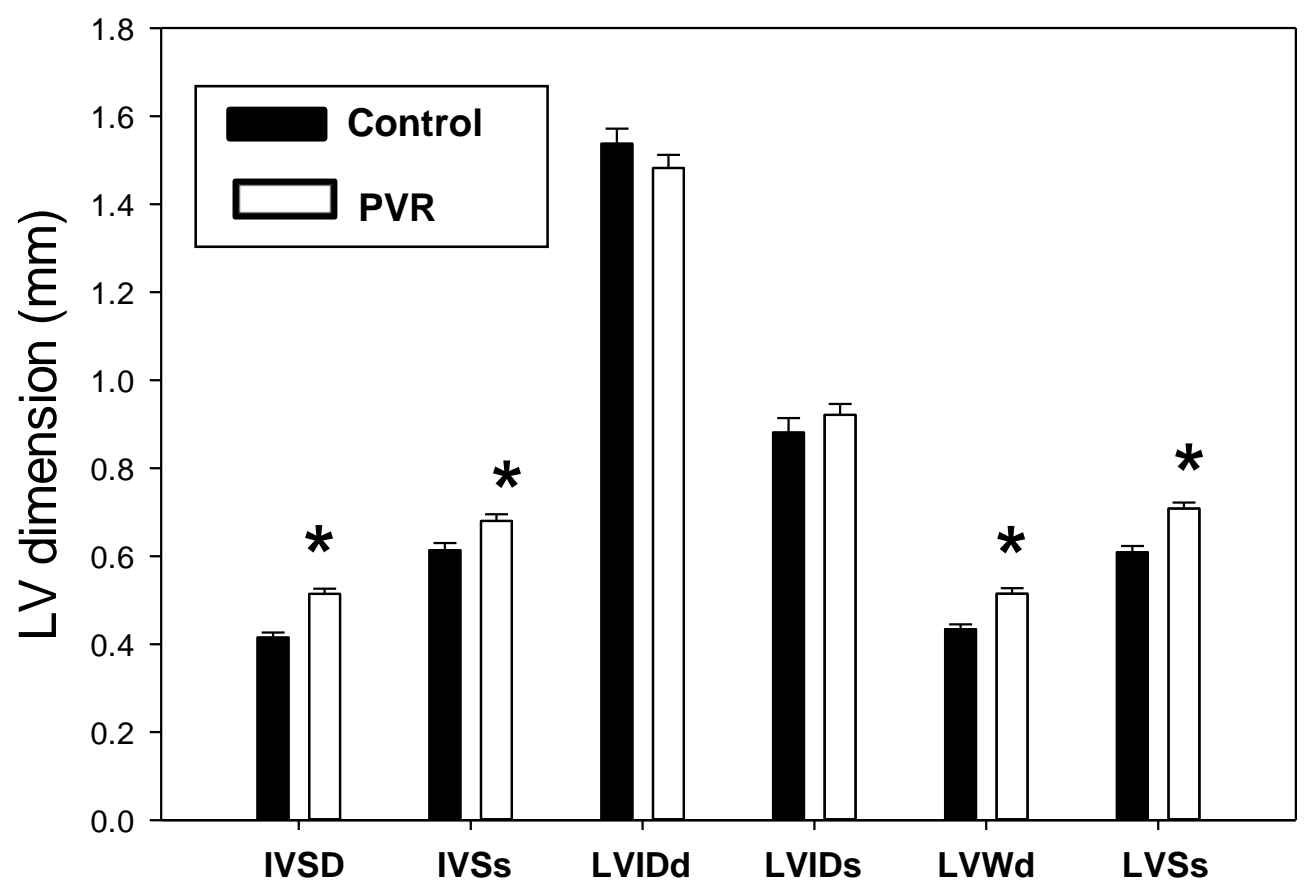

B

C
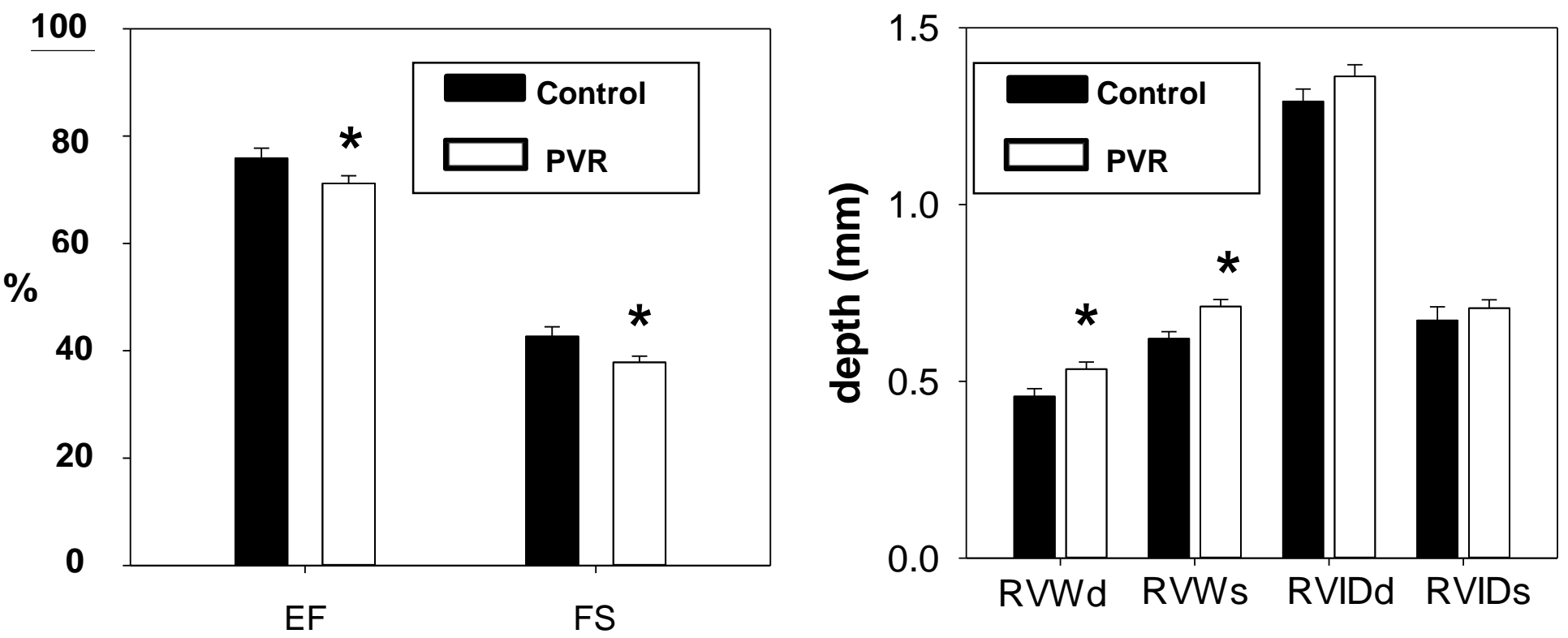
Figure 4

A

B

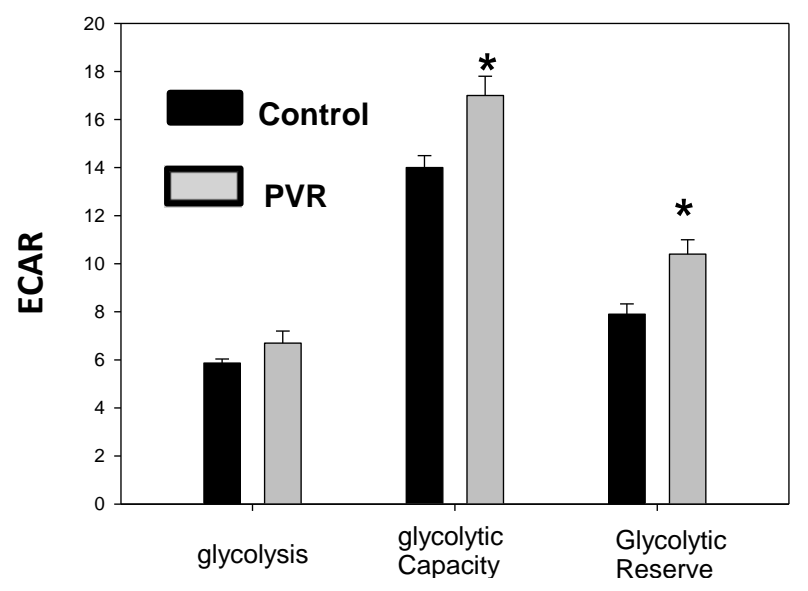

C

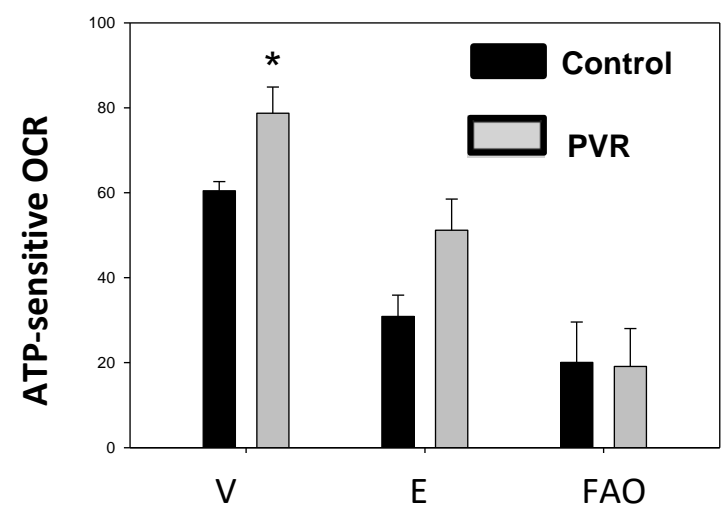

E

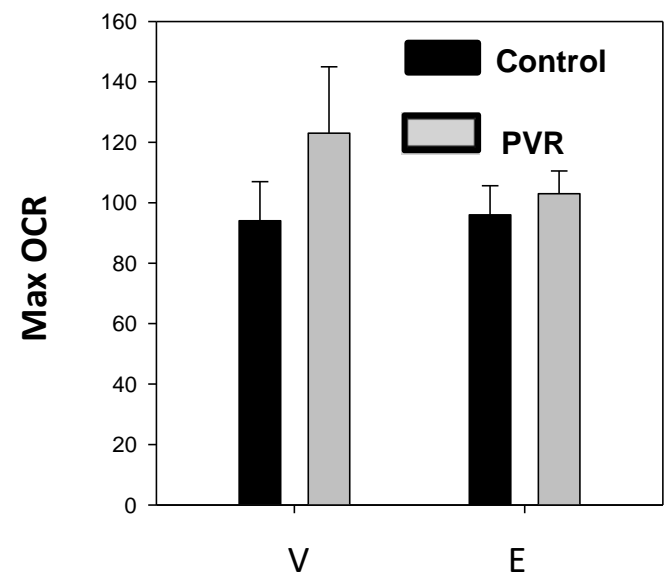

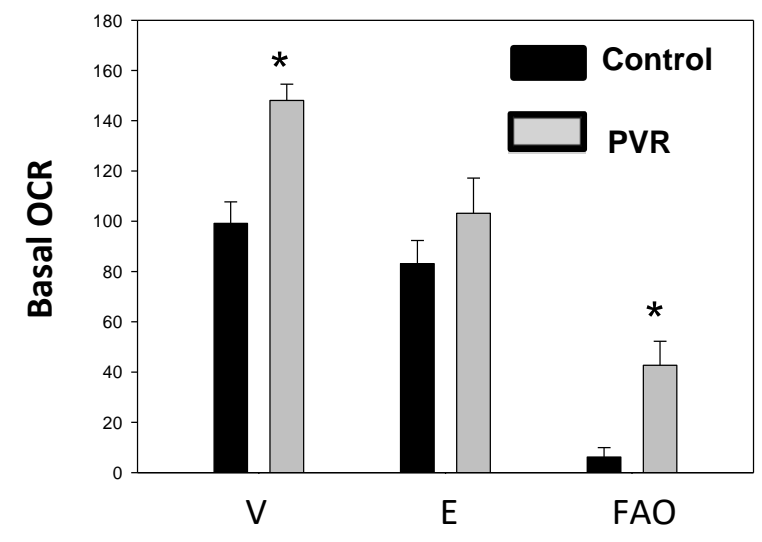

D

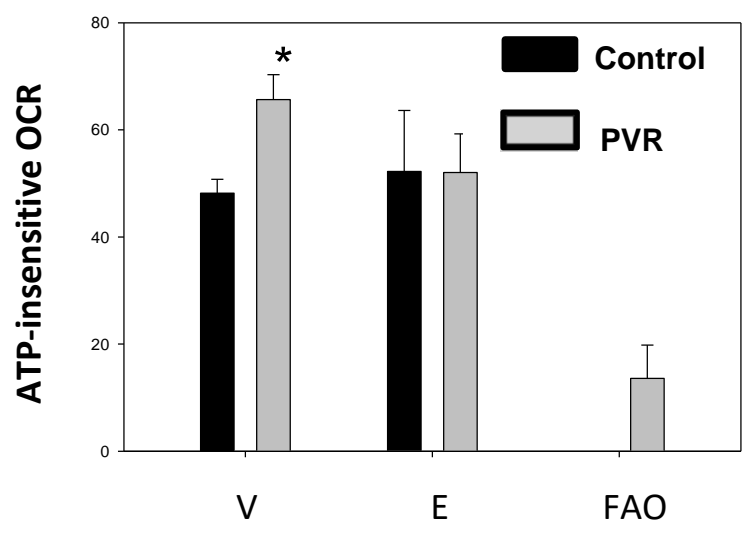


Figure 5

A
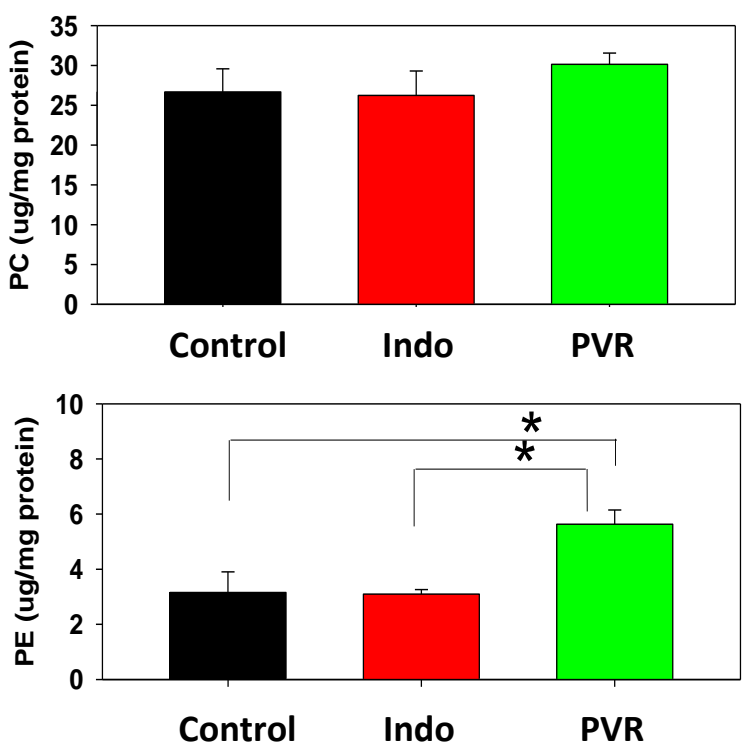

D

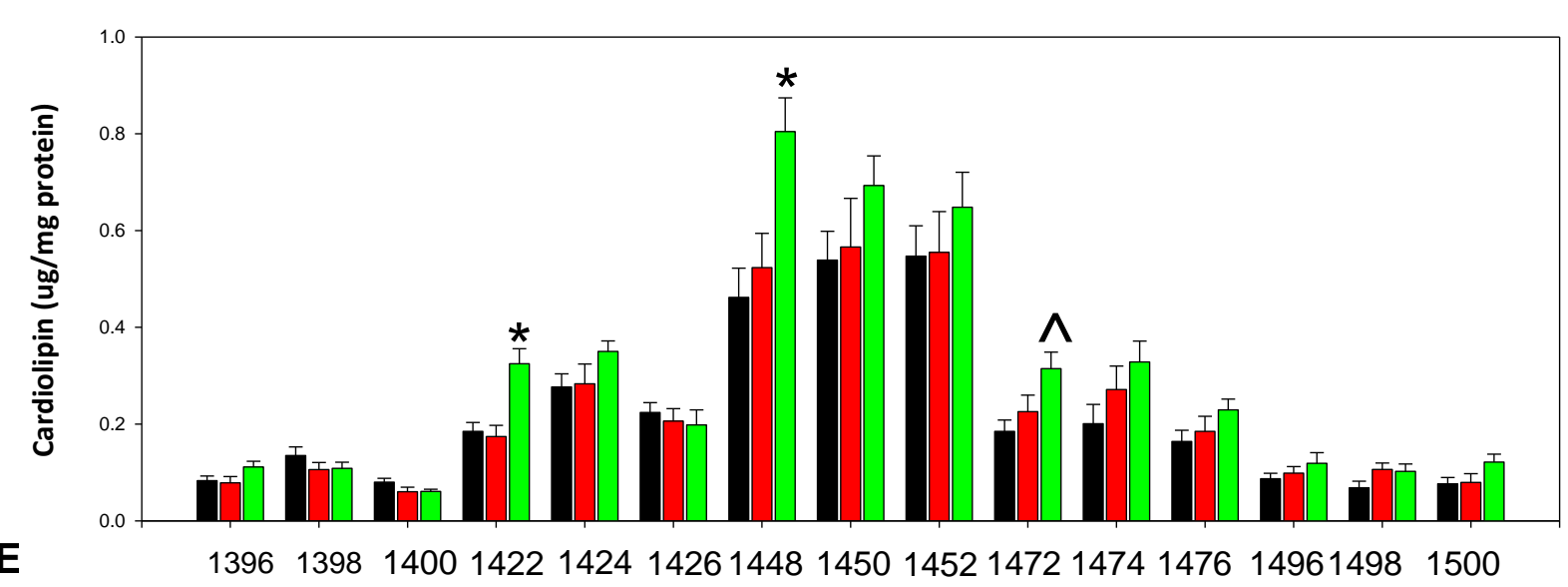

$\mathbf{E}$

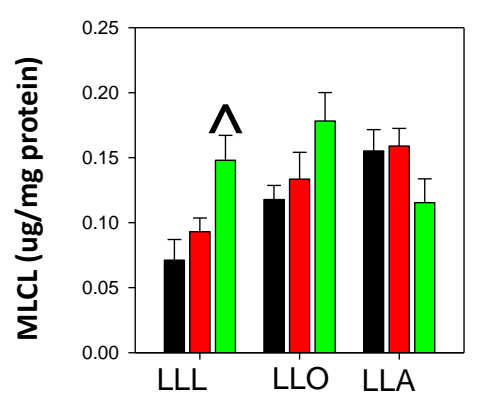

B

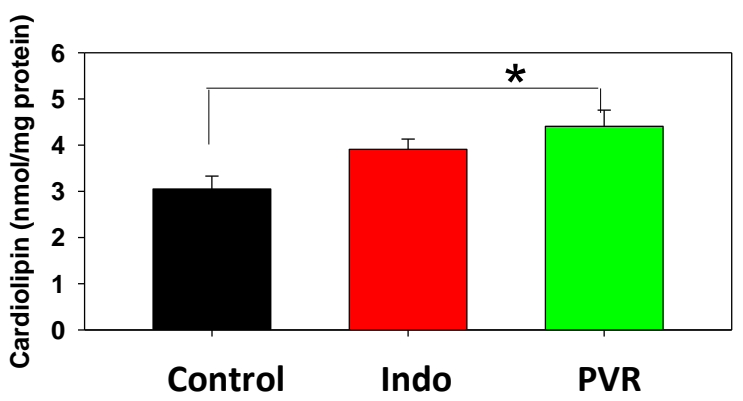

\section{D}




\section{Figure 6}

A
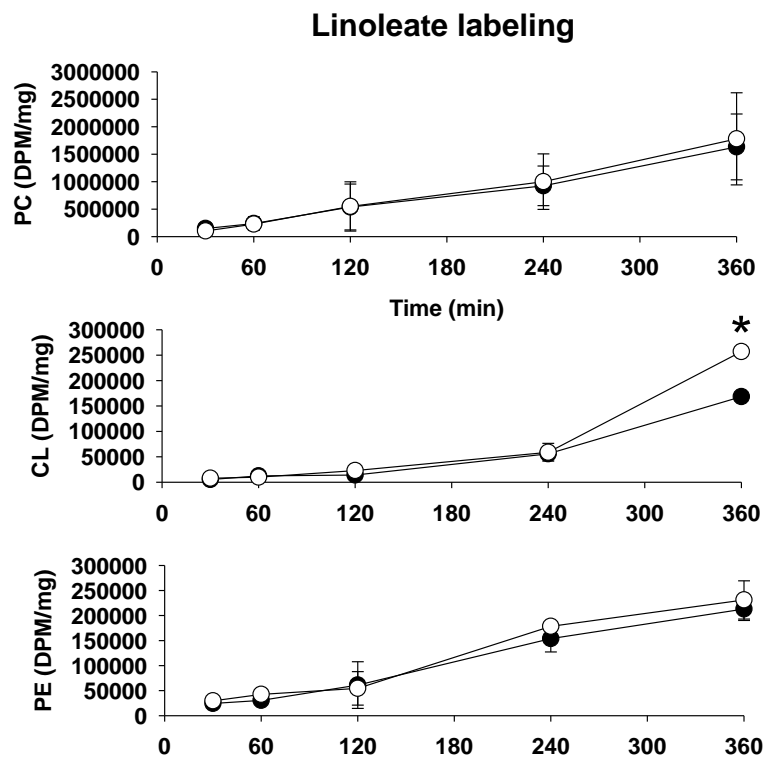

C
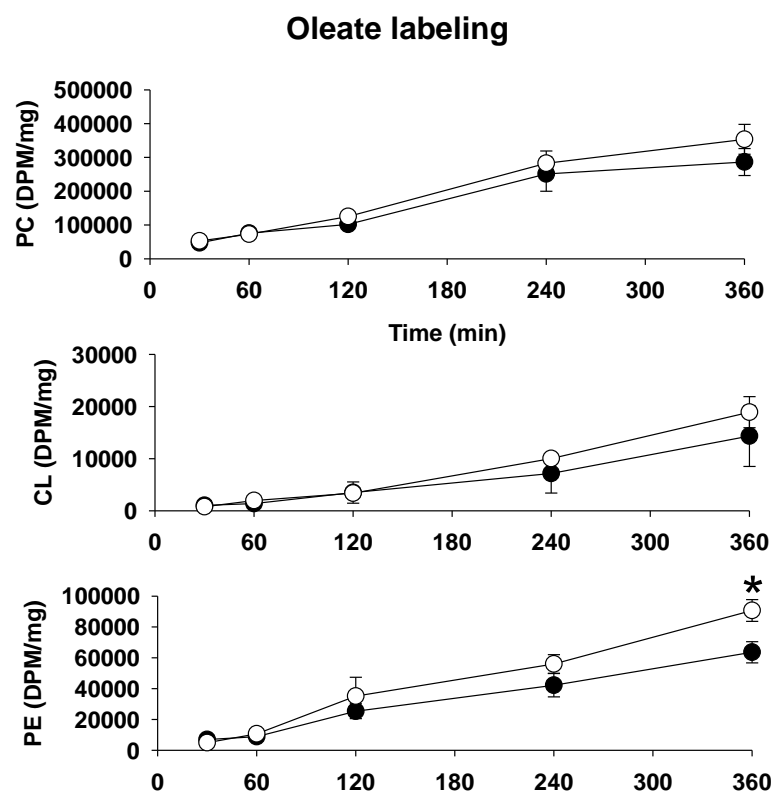

B

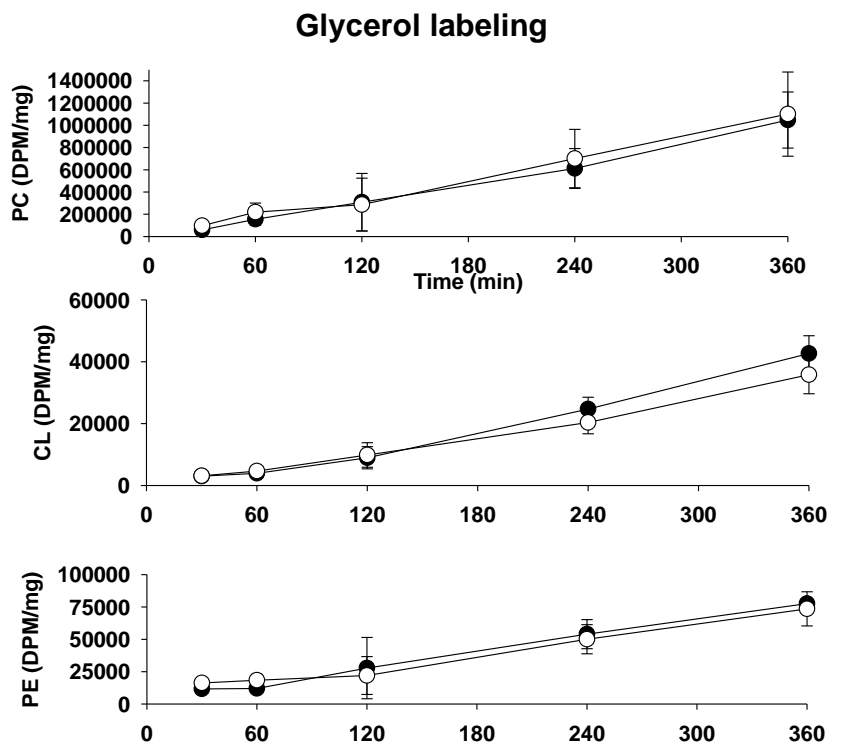


Supplementary Figure 1. A. Mitochondrial oxygen consumption rate $(\mathrm{OCR})$ in isolated control and PVR cardiac myocytes with glucose as substrate. B. Content of respiratory complexes in mitochondrial fractions from adult rat heart mitochondria and from control and PVR hearts. $\alpha$-Tubulin is shown as loading control and individual respiratory complexes are indicated on the left. $n=3-4$.

A

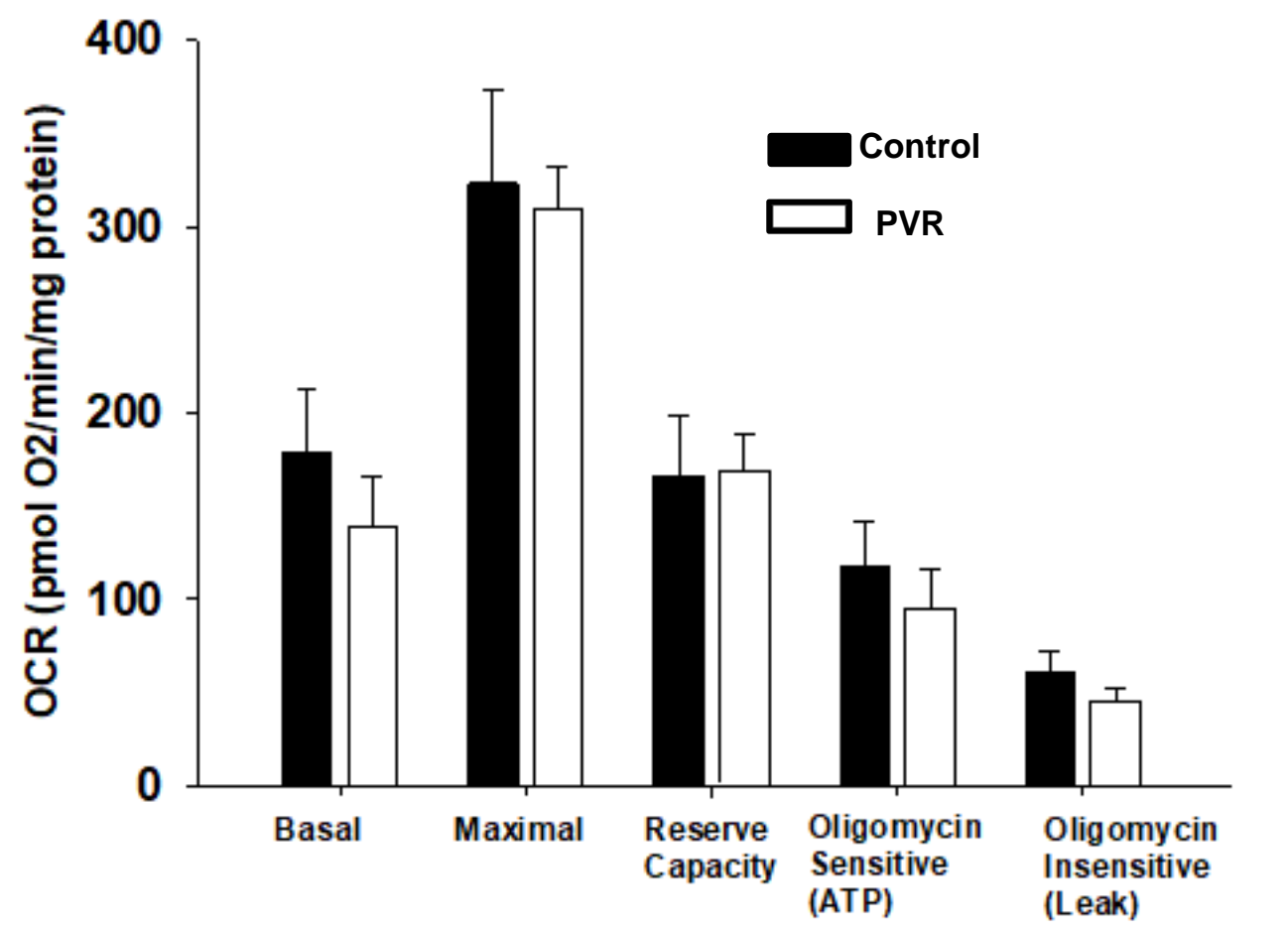

B

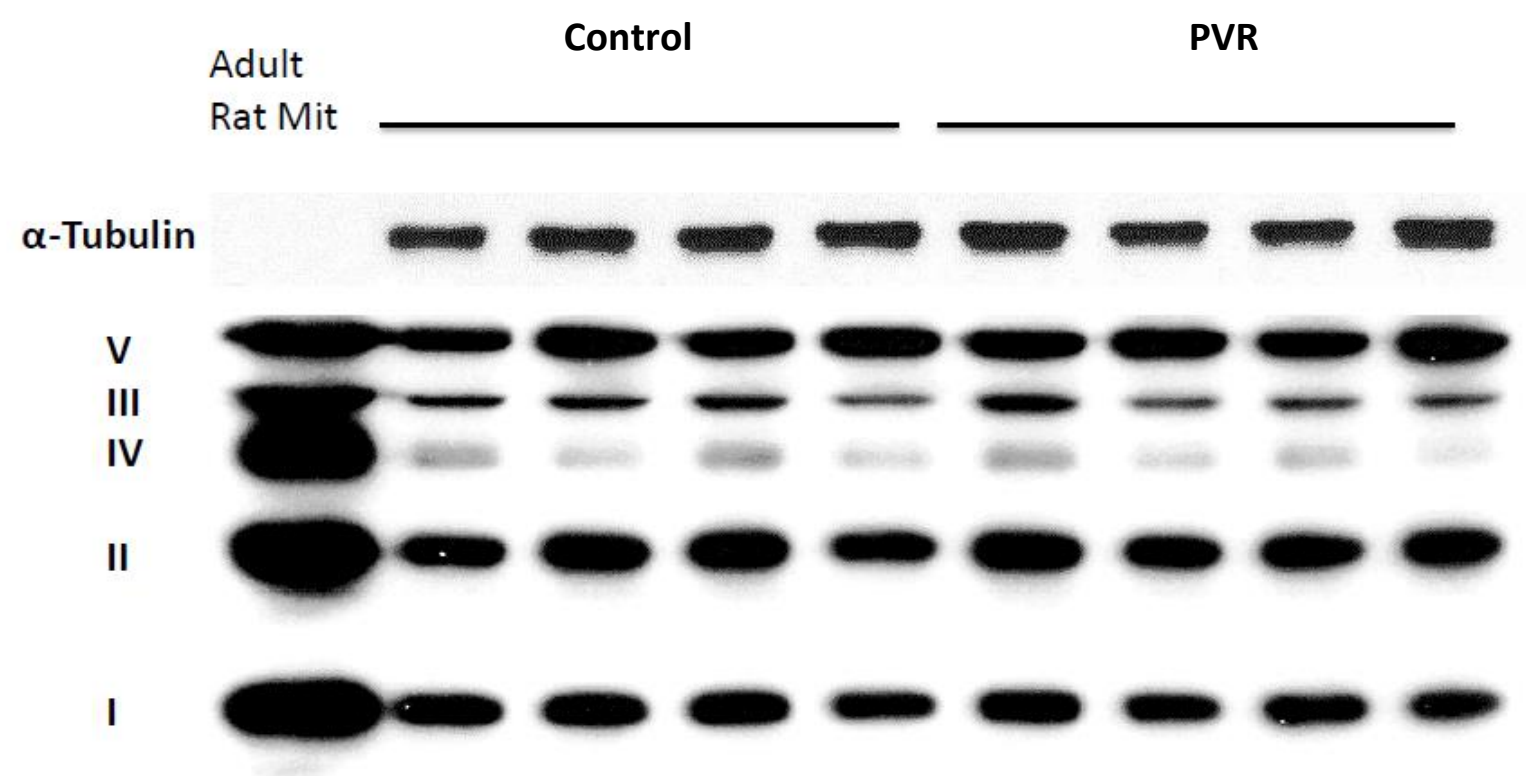


Supplementary Figure 2. A. Cholesterol ester (CE); B. Triacylglycerol (TG); C. Free cholesterol (FC) in hearts of newborn pups from control, indomethacin (Indo) or PVR rats. $n=3$.

A

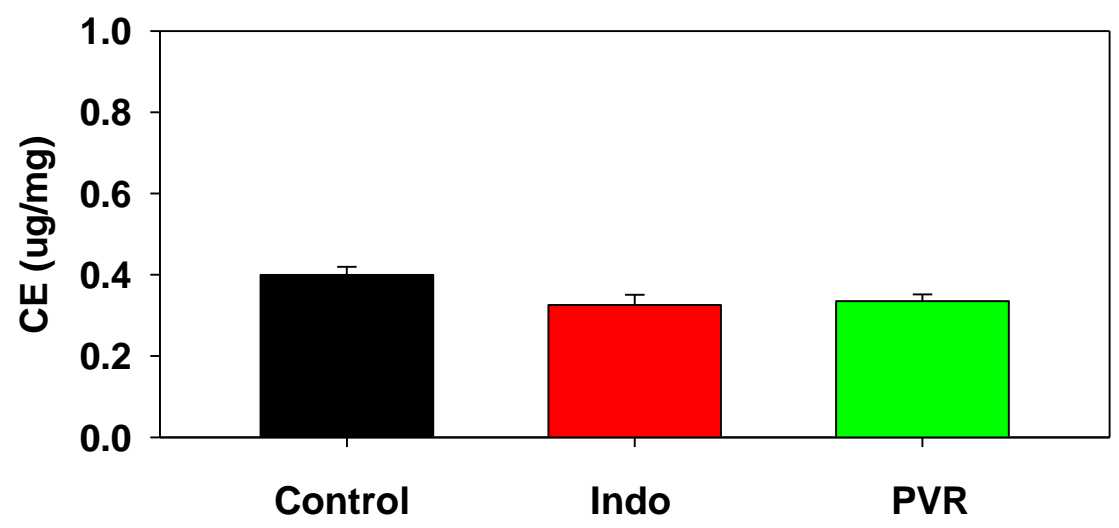

B

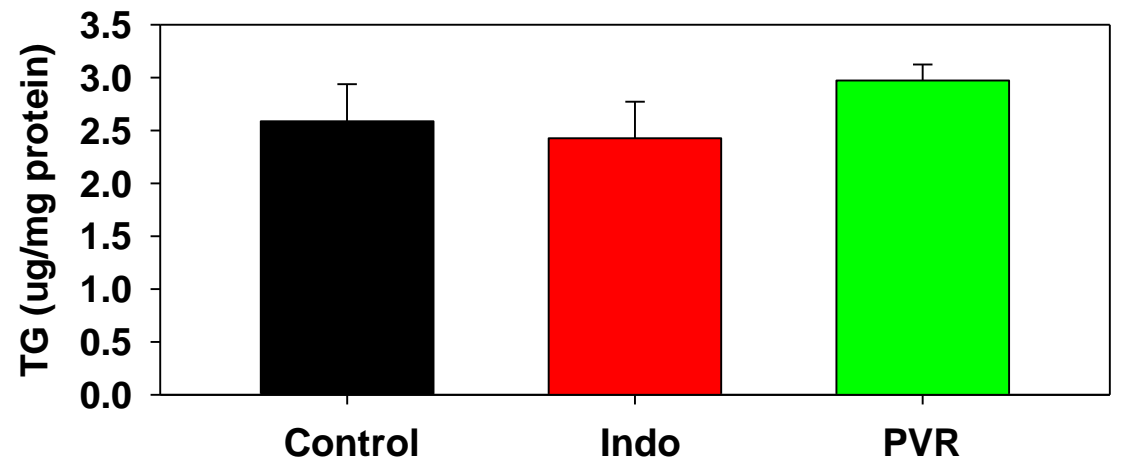

C

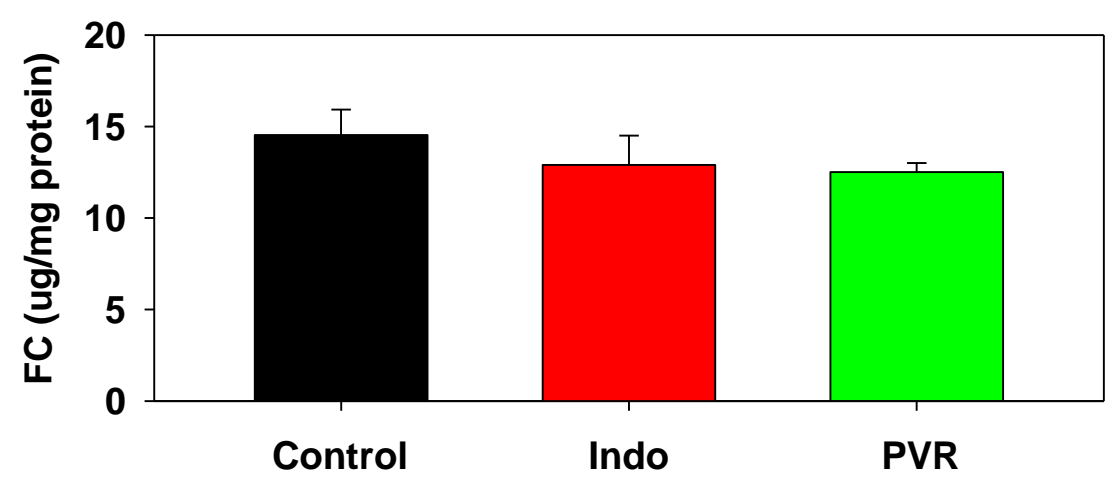


Supplementary Figure 3. Synthesis of PE from ethanolamine or serine. A. Incorporation of $\left[{ }^{3} \mathrm{H}\right]$ ethanolamine into PE in isolated control and PVR cardiomyocytes. B. Incorporation of $\left[{ }^{3} \mathrm{H}\right]$ serine into phosphatidylserine (PS) or into PE in isolated control and PVR cardiomyocytes. $n=3$.

A

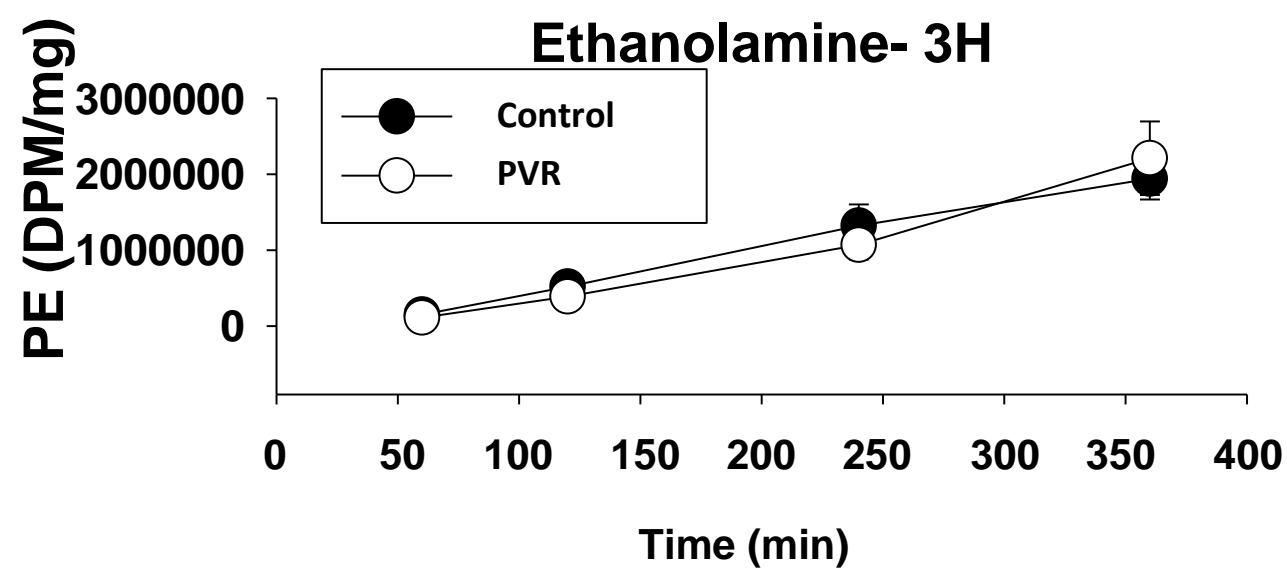

B

Serine - 3H

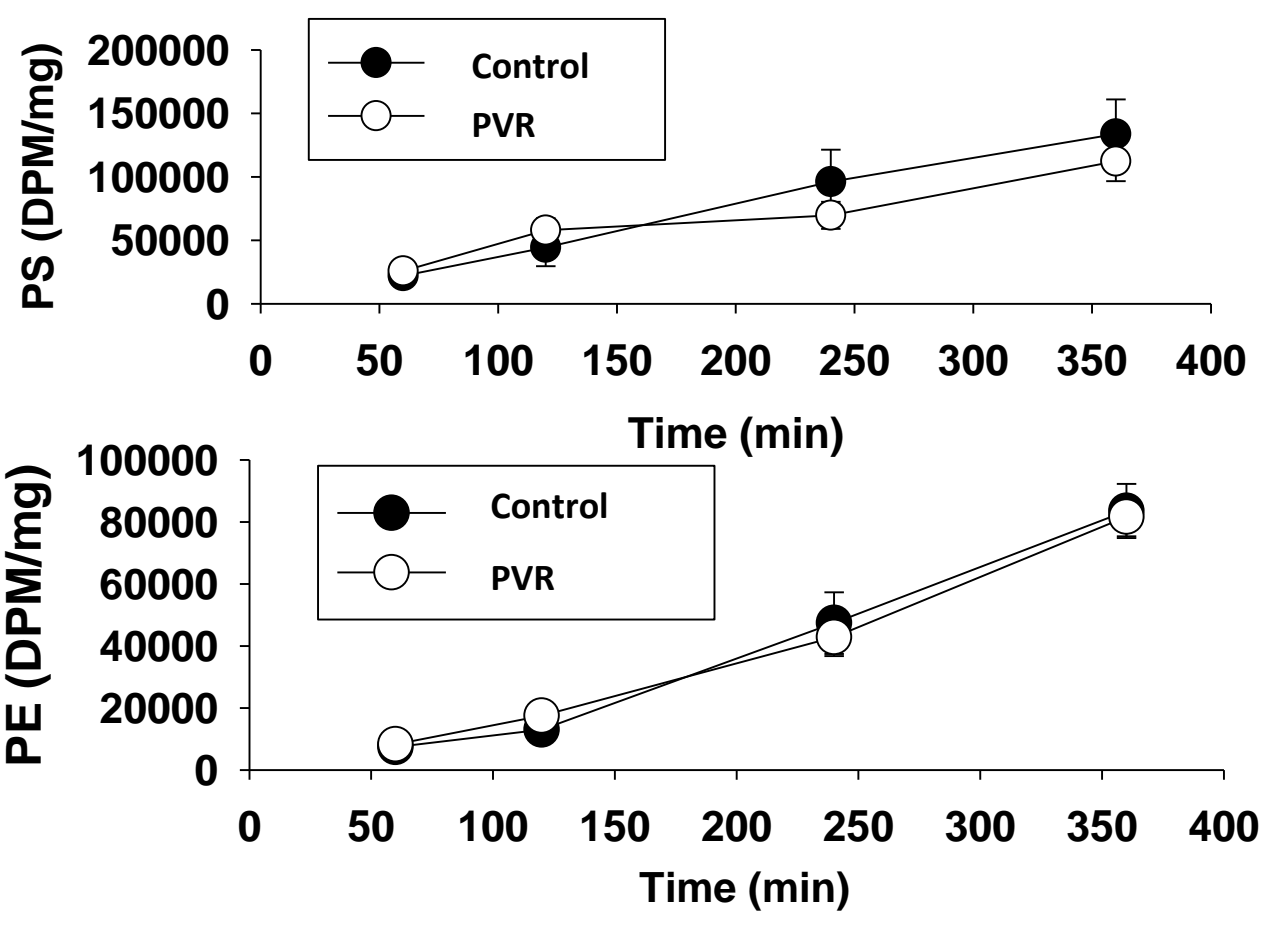

